\title{
Le rythme et la voix, premiers défis d'un traducteur de Claude Simon
}

Karin Holter

\section{OpenEdition}

1 Journals

Édition électronique

URL : http://journals.openedition.org/ccs/940

DOI : $10.4000 /$ ccs. 940

ISSN : 2558-782X

Éditeur :

Presses universitaires de Rennes, Association des lecteurs de Claude Simon

\section{Édition imprimée}

Date de publication : 30 avril 2015

Pagination : 21-30

ISBN : 9782753539990

ISSN : 1774-9425

Référence électronique

Karin Holter, "Le rythme et la voix, premiers défis d'un traducteur de Claude Simon », Cahiers Claude Simon [En ligne], 10 | 2015, mis en ligne le 22 septembre 2017, consulté le 21 décembre 2020. URL http://journals.openedition.org/ccs/940 ; DOI : https://doi.org/10.4000/ccs.940 


\title{
LE RYTHME ET LA VOIX, PREMIERS DÉFIS D'UN TRADUCTEUR DE CLAUDE SIMON
}

\author{
Karin HOLTER \\ Université d'Oslo
}

Pour tout lecteur il y a des rencontres textuelles plus décisives que d'autres. À plus forte raison pour un étudiant ou doctorant, puisque cela peut déterminer la direction de sa vie professionnelle. Cela a été mon cas, à ma première lecture de la première phrase de La Route des Flandres:

Il tenait une lettre à la main, il leva les yeux me regarda puis de nouveau la lettre puis de nouveau moi, derrière lui je pouvais voir aller et venir passer les taches rouges acajou ocre des chevaux qu'on menait à l'abreuvoir, la boue était si profonde qu'on enfonçait dedans jusqu'aux chevilles mais je me rappelle que pendant la nuit il avait brusquement gelé et Wack entra dans la chambre en portant le café disant Les chiens ont mangé la boue, je n'avais jamais entendu l'expression, il me semblait voir les chiens, des sortes de créatures infernales mythiques leurs gueules bordées de rose leurs dents froides et blanches de loups mâchant la boue noire dans les ténèbres de la nuit, peut-être un souvenir, les chiens dévorants nettoyant faisant place nette: maintenant elle était grise et nous nous tordions les pieds en courant, en retard comme toujours pour l'appel du matin, manquant de nous fouler les chevilles dans les profondes empreintes laissées par les sabots et devenues aussi dures que de la pierre, et au bout d'un moment il dit Votre mère m'a écrit.

Étonnement et enthousiasme devant la circularité de cette phrase, de son pouvoir visuel et englobant, de sa diversité. C'est une phrase qui, de façon très précise nous présente, dans son contexte réel et imaginé, une scène du passé remémorée, une scène qui nous est racontée par une voix narrative qui se rappelle, qui imagine et réfléchit, qui nous rend présent le passé: "maintenant elle était grise ». Loin de moi, à l'époque, de penser à la traduction. Et en fait, techniquement, cette phrase typiquement simonienne ne pose pas de gros problèmes, du moins dans ma langue. Mais elle pose trois questions 
fondamentales auxquelles tout traducteur de Claude Simon doit répondre: la question du mouvement du texte, de son rythme; la question de la voix; et enfin, la question de la force génératrice des mots.

\section{PREMIERS DÉFIS}

Premiers, dans mon titre, veut dire "les plus importants", mais aussi ce à quoi un traducteur de Claude Simon est confronté d'abord et tout de suite, dès la première phrase, le premier paragraphe. Il y a d'autres défis, bien sûr, auxquels nous devons faire face, des défis techniques dus à l'organisation interne de nos langues respectives par rapport au français, des défis factuels et culturels - et ils sont considérables - mais si nous n'arrivons pas à rendre, à transférer dans notre langue à nous le rythme et la voix du texte simonien, c'est raté, et cela dès la première phrase. Oui, on ne saurait trop souligner l'importance de l'incipit, des premiers mots d'un texte; ils sont comme une sorte de résumé très condensé - thématique, structural et tonal - de tout ce qui va être développé ensuite. Qu'il nous suffise de citer ici le grand critique Jean Starobinski évoquant le début du Neveu de Rameau de Diderot:

Les premières lignes du texte, comme toutes les ouvertures, méritent une attention extrême. Car si les "principes " dont je viens de parler sont véritablement à l'œuvre dans Le Neveu de Rameau ils doivent être décelables dès le commencement du texte; celui-ci devrait être d'entrée de jeu sous leur dépendance ${ }^{1}$.

L'ouverture du texte est donc un lieu privilégié d'encodage pour l'écrivain, pour le critique c'est un lieu privilégié d'observation, et pour le simple lecteur, sa première rencontre affective et intellectuelle avec un univers textuel.

Difficile dans ce cas de sous-estimer le rôle du traducteur pour faire passer dans sa langue à lui la tonalité et l'information contenue dans un début de roman et, à plus forte raison, son importance pour la réussite ou la non-réussite d'un écrivain à traverser les frontières et s'implanter dans d'autres langues, pour d'autres lecteurs. Il y a des écrivains frustrés, comme Kundera qui, après avoir lu ses textes en traduction française, décide de les traduire lui-même. Pour Beckett, la question ne se posait même pas: il se traduisait lui-même, dans les deux sens, ou plutôt, il présentait deux versions, française et anglaise, de ses œuvres, tenant toujours compte du contexte spécifique culturel et linguistique de chaque pays. Claude Simon, lui, a eu de la chance; aussi ses premiers mots, à la récep-

1. Jean Starobinski, "L'incipit du Neveu de Rameau », La Nouvelle Revue Française, décembre 1981. 
tion du Prix Nobel, ont été pour Bjurström, son traducteur suédois. Carl Gustaf Bjurström, éminent critique et présentateur de l'œuvre aussi bien que traducteur et qui reste pour nous, traducteurs en langues scandinaves de Simon, un modèle ${ }^{2}$.

\section{LA FIDÉLITÉ AU TEXTE}

Fondamentalement, et surtout pour ce qui est du rythme, c'est de cela qu'il s'agit, de fidélité. Anne-Lisa Amadou, notre traductrice de Proust durant 30 ans, parle de setningsmelodien, la mélodie de la phrase comme la première exigence imposée à un traducteur de la Recherche ${ }^{3}$. Pour le rythme et la construction de la phrase, Proust et Simon posent des défis semblables au traducteur. Comme pour Proust, il faut essayer, encore et encore, de faire passer dans notre langue le mouvement, le tempo, lent ou rapide de la phrase simonienne. Concrètement, comme ce sont les phrases longues qui posent surtout problème pour un traducteur norvégien, la première consigne est de ne pas les couper, mais de les "faire durer" aussi longtemps que chez Simon, de suivre la poussée de leur souffle jusqu'au bout, quitte à bousculer les règles de "bonne conduite » de la langue norvégienne. Mais, nous le savons, Simon aussi a passablement bousculé ces règles, assez pour être puni par le jury de Goncourt, à propos de La Route des Flandres, pour "son mépris de la ponctuation française".

Et dans la pratique, il s'agit justement souvent de ponctuation : à éviter donc le point final s'il ne se trouve pas dans le texte original; il faut le remplacer, quand le souffle et le sens le commandent, par des virgules, points-virgules et autres points, ou par la reprise d'un même mot; essayer, dans une langue comme le norvégien qui n'a pas la chance de pouvoir employer les participes présents comme le français ou l'anglais, de trouver des conjonctions, des formes verbales, des adjectifs qui puissent fonctionner à peu près de la même façon. Garder toujours les constructions tripartites! Lire à haute voix le texte dans les deux langues pour tester si ça colle. La pratique du gueuloir de Flaubert doit être obligatoire aussi pour nous, traducteurs. En conclusion, il faut se battre avec sa propre langue afin de la rendre plus malléable.

2. Il y aurait beaucoup à dire sur la condition de travail des traducteurs. Pour un traducteur professionnel il faut traduire vite pour gagner sa vie. Si Claude Simon a eu de la chance, c'est aussi parce que beaucoup de ses traducteurs ont été des " fidèles " qui ont suivi sa carrière d'un bout à l'autre et qui ont pu se permettre de prendre le temps qu'il faut, travaillant con amore puisque leur gagne-pain est assuré par un autre emploi, par exemple dans l'édition ou dans une université.

3. Anne-Lisa Amadou, « Når språket blir mimetisk », dans Det umuliges kunst, Réd. Per Qvale, Oslo, Aschehoug, 1991, p. 87. 
Anne-Lisa Amadou, en commentant son travail, a dit aussi ceci, et je la cite de mémoire: "Un texte traduit de Proust ne doit pas avoir l'air d'être écrit par un écrivain norvégien; il doit montrer la langue norvégienne après qu'elle a été exposée à une rencontre drastique avec une langue étrangère. " Cela vaut aussi pour la traduction de Claude Simon. Avec la différence qu'aujourd'hui, grâce à ces rencontres "drastiques " entre les deux langues, le norvégien est devenu plus malléable, plus extensible et qu'on peut trouver des écrivains de chez nous, et parmi les plus grands ${ }^{4}$, qui écrivent un norvégien dans le sillage de Proust et Simon.

\section{LA LENTEUR DU DÉVOILEMENT}

Quant au rythme simonien, ce qui le crée, est une construction de la phrase ou de la description qui dévoile lentement, pas à pas, son objet. C'est dire que, dans la traduction aussi, il faut essayer de garder le mot de la fin pour la fin. Dans l'incipit de La Route des Flandres, par exemple, laisser les chevaux se matérialiser à partir des taches de couleurs; dans l'ouverture d'Histoire, si possible, éviter de nommer le référent d'elles dans "l'une d'elles » avant qu'il n'apparaisse, au fond d'une parenthèse: "dans l'obscur fouillis des branches".

Si possible! Il y a évidemment des limites dans la pratique de la fidélité au texte qu'on va traduire. Il faut aussi être fidèle à sa propre langue, on ne fait pas du mot à mot quand on traduit. Il faut que le son reste norvégien, que l'ordre des mots et la syntaxe soient respectés et que la phrase, même étirée, doublée ou triplée en volume - ou coupée au milieu - retombe sur ses pieds norvégiens. N'empêche que dans la discussion entre théoriciens de la traduction qui oppose ceux qui préconisent l'importance de la langue source et ceux qui donnent la priorité à la langue cible, vous l'aurez compris, j'opte pour les «sourciers».

\section{«L'EFFET DE VOIX »}

C'est ainsi que Dominique Rabaté intitule le premier chapitre de son livre Vers une littérature de l'épuisement ${ }^{5}$. En présentant quelques débuts de romans, celui de La Chute, D'un château l'autre et de L'Innommable, il constate " qu'une grande partie des œuvres contemporaines pourrait porter comme sous-titre cette phrase de Jean Cayrol, "On vous parle" ». L'effet de voix, c'est justement l'impact sur le

\footnotetext{
4. Je pense en premier lieu à Ole Robert Sunde (1952-), Rune Christiansen (1963-), Anne Oterholm (1964-) et Christopher Grøndahl (1969-). Voir aussi « Paroles d'écrivains », Cahiers Claude Simon, Presses Universitaires de Perpignan, n 6, 2010.
}

5. Dominique Rabaté, Vers une littérature de l'épuisement, José Corti, 1991. 
lecteur de la présence d'une voix qui, de Proust et Faulkner à Simon, de Camus et Céline à Pinget et Beckett, caractérise certains textes $\mathrm{du} \mathrm{xx}^{\mathrm{e}}$ siècle éminemment écrits. D'où la nature paradoxale de ces textes; personne ne parle "réellement " comme ces narrateurs, mais cette simulation d'oralité a comme effet de nous impliquer, nous lecteurs, dans ce qui semble une adresse directe ${ }^{6}$.

Si la présence vocale est manifeste dans tous ces romans, il faut souligner aussi la différence de tonalité qui caractérise la ou les voix de ces narrateurs. Chez Beckett, c'est la même voix, unique et répétitive de livre en livre, un soliloque, une voix qui tout en nous impliquant parle surtout à elle-même. Chez Simon aussi, nous trouvons cette voix qui s'interroge, qui ne sait pas: "mais je me rappelle ", "Mais comment savoir?". Au "Comment c'est " beckettien répond le "Comment était-ce?" simonien. Seulement, chez Simon, ce qui nous frappe d'emblée, et qui pose d'autant plus de défis au traducteur, c'est la pluralité de voix, son univers fortement dialogique. D'abord à cause de la présence massive dans ses romans d'autres voix que celle qui porte la narration, ensuite, parce que la voix narrative simonienne elle-même se transforme en cours de route, quelquefois de façon dramatique, pouvant changer complètement de registre d'un roman à l'autre, quelquefois même à l'intérieur du même roman (comme à la fin de $L a$ Bataille de Pharsale). Grosso modo, on peut distinguer trois étapes - ou registres de la voix narrative chez Simon: celle, subjective, interrogative et passionnée qui va du Vent à La Bataille de Pharsale; celle, neutre et objectiviste, toujours au présent des " romans-descriptions " des années 70 ; celle, des Géorgiques au Tramway, qui use de tous les registres.

Donc, nous aussi, en tant que traducteurs, nous avons besoin de tous nos registres pour trouver à chaque fois le ton juste. D'autant plus qu'en dehors d'une voix narrative variable, nous avons à faire face aussi à beaucoup de discours de personnages, dont deux traversent d'un bout à l'autre l'œuvre simonienne. C'est d'abord la voix, emblématique, qui ouvre Le Vent:

Un idiot. Voilà tout. Et rien d'autre. Et tout ce qu'on a pu raconter ou inventer, ou essayer de déduire ou d'expliquer, ça ne fait que confirmer ce que n'importe qui pouvait voir du premier coup d'œil. Rien qu'un simple idiot.

6. Ceux et celles qui ont eu le privilège d'assister au premier séminaire sur le Nouveau Roman à Cerisy-la-Salle en 1971, le haut lieu du scriptural, se rappellent la "gêne " qui a suivi l'intervention de Robert Pinget qui a proclamé que l'intérêt de son travail " a été la recherche d'un ton ", qu'il n'assumait que " des erreurs de ton " et que "seule capte [son] intérêt la voix de celui qui parle ». C'est que l'heure - théorique/ idéologique n'était pas encore venue pour la notion de voix et d'écoute. Et cela malgré Pinget, Beckett, Sarraute et Simon! Robert Pinget, "Pseudo-principes d'esthétique » dans Nouveau Roman : Hier, aujourd'hui, tome 2 : Pratiques (actes du Colloque de Cerisy, 20-30 juillet 1971), Paris, UGE, coll. « 10/18 », 1972, pp. 311-324. 
C'est la voix de l'affect et de la hargne couplés avec un certain plaisir dans la formulation des propos ironiques, cyniques, insultants. C'est la voix des " forts " ou qui voudraient se montrer tels: forts en paroles, en préjugés, en expérience vécue, ici le notaire, ailleurs Blum dans La Route, l'Américain du Palace, parfois l'oncle... La cible de leur attaque verbale est souvent le personnage central du roman, qui lui ne trouve pas ses mots, qui ne maîtrise rien: Montès, Georges, le soldat... Mais plus généralement, c'est l'Histoire ou la vie elle-même qui est mise en accusation par ces maîtres-parleurs que nous, les traducteurs, devons faire parler de la même façon dans notre langue.

Une autre voix caractéristique résonne de part et d'autre dans l'univers simonien, celle, outragée, de la plainte, de la révolte impuissante, celle qui parle pour peupler le silence ou forcer l'écoute de l'autre, celle de la " mère " de L'Herbe et $L a$ Route mais aussi "la voix usée " du vieux général des Géorgiques: " un monotone ressassement de griefs, de reproches, et à la fin, s'exhalant, s'étirant, interminable, désolée, morne, une plainte». Et toujours, dans ce registre, la voix de la " grandmère ", " la même voix geignarde plaintive " qui ne cesse de hanter les lieux de la fiction simonienne. Deux voix de personnages contrastées donc, mais toutes deux chargées d'émotivité, créant la même impression de présence phatique.

\section{LA JUSTESSE DE TON}

Il y a dans la caractérisation des voix de personnages chez Simon une telle justesse de ton, une telle sûreté de simulation quant au débit, l'intonation, l'appartenance sociale des " usagers » qu'il est évident que l'écrivain qui les met en place prend plaisir lui-même à jouer avec toutes les ressources de la langue orale. Mais si la caractérisation des voix remplit, la plupart du temps, une fonction sociologique ou psychologique, donc " classique ", il arrive, comme dans les deux "Divertissement " de Leçon des choses, qu'importe surtout le besoin de variation, de changement de registre, de ton; très concrètement peut-être, le besoin de l'écrivain d'une note nouvelle pour (nous/se) divertir.

Chaque fois que je relis ces pièces de bravoure, la réécriture burlesque, tragi-comique, féroce de certains épisodes clés de La Route des Flandres, je ris aux éclats, me disant en même temps, "ça, je ne saurai jamais le traduire! » Et pourtant, il faudrait tenter de le faire, certains l'ont fait, ont peut-être réussi à rendre dans leur propre langue ce timbre-là. Alors chapeau! Un autre problème se pose, cependant, à propos de ce type de textes et qui concerne l'écrivain aussi bien que le traducteur: leur inscription dans le temps. Liée à une époque et à un milieu 
social spécifique, la langue orale, populaire et argotique employée par tel écrivain dans des parties dialoguées ou monologuées risque d'être incompréhensible pour une nouvelle génération de lecteurs. Alors que faire? Laisser au texte sa date ou le "moderniser »? Remplacer «l'accent faubourien » du petit juif (Blum) par l'accent arabe des banlieues ${ }^{7}$ ?

LA FICTION MOT À MOT

Je ne connais pour ma part d'autres sentiers de la création que ceux ouverts pas à pas, c'est-à-dire mot après mot, par le cheminement même de l'écriture.

Cette phrase, si souvent citée de la préface d'Orion aveugle, nous met en tant que traducteurs de Claude Simon face à un troisième défi, évident, formidable, mais auquel chacun doit répondre avec les ressources de sa propre langue, à savoir l'importance des mots eux-mêmes comme générateurs de fiction. Si nous nous reportons encore à l'ouverture de La Route des Flandres, nous remarquons que plus d'un tiers du texte découle de l'expression " jamais entendue ", Les chiens ont mangé la boue. "Il me semblait voir » : la force visuelle, la dimension mythique, la construction tripartite - « les chiens dévorants nettoyant faisant place nette »-, la voix au présent, " peut-être un souvenir » du narrateur, de l'écrivain au travail; à

7. Il est difficile de discuter les problèmes de la traduction sans soulever aussi la question de la retraduction. Vu l'importance d'une bonne traduction pour la possibilité d'une ouvre littéraire de s'implanter dans une autre langue, il faudrait sans doute retraduire beaucoup plus souvent. Difficile cependant de persuader un éditeur de repayer pour une traduction déjà faite. À moins que, à propos d'une réimpression, on ne se rende compte de fautes ou de coupures trop évidentes. Comme quand on a découvert que le premier traducteur en norvégien de Madame Bovary avait sauté la scène du fiacre avec Emma et Léon à travers Rouen, ou, plus grave et plus près de nous, l'omission dans la traduction danoise de La Modification de toutes les séquences de rêves... Mais dans ces cas, on parle plutôt d'une révision de la traduction antérieure. Et pourtant, le destin de tous les grands classiques - la Bible, Dante, Shakespeare, Goethe, Dostoïevski, et pour le centenaire de Swann, Proust - c'est d'être régulièrement retraduits. Comme si le texte original survivait toujours à sa traduction, comme si, dans chaque génération ou chaque siècle, de nouveaux lecteurs et traducteurs découvraient de nouveaux aspects de l'œuvre. Quelquefois, c'est le développement de la langue écrite d'un pays qui exige des traductions nouvelles. C'est le cas du norvégien qui se transforme, surtout en ce qui concerne le vocabulaire et l'orthographe, beaucoup plus vite que le français. La raison historique en est notre union avec le Danemark (1380-1814). En 1814, la langue officielle écrite en Norvège était donc le danois, mais qui, sous l'influence de la langue parlée (surtout dans les villes) et de beaucoup de réformes de langue est devenu le norvégien - bokmål - que la majorité des Norvégiens écrivent aujourd'hui. Grâce au linguiste et écrivain Ivar Aasen (1813-1896), nous avons une deuxième langue officielle en Norvège, le néo-norvégien/nynorsk, construit à partir de certains dialectes spécifiques, le landsmål, (la langue des campagnes). En Norvège, le marché de la traduction est donc double: Racine et Beckett sont lus et joués dans les deux langues. Et comme les deux langues évoluent, se rapprochant, la question de la nécessité de retraduire les classiques tous les 50 ans vient d'être soulevée par l'Association des traducteurs norvégiens. 
partir de cette expression nous sont présentés, en résumé, « les principes " mêmes qui seront à l'œuvre dans La Route des Flandres. C'est comme si l'écrivain, tout au début de son roman, nous lançait un "Attention aux mots! ", qu'il nous offrait une leçon de lecture.

La première fois que l'ambiguïté ou la polysémie de la langue française nous saute aux yeux - intraduisible - dans ce roman est sans doute (à propos du capitaine de Reixach): "ces réflexes et traditions conservés comme qui dirait dans la Saumur " (saumure). Mais s'il faut renoncer devant de tels cas, le traducteur pourra - et devra - compenser cette perte ailleurs dans le texte; l'essentiel c'est que, dans la traduction aussi, la langue montre son nez, nous force à voir sa force génératrice. La bifurcation opérée une page plus loin dans La Route (à propos de la mort du capitaine) est plus facilement traduisible:

Un instant l'éblouissant reflet de soleil accroché ou plutôt condensé, comme s'il avait capté attiré sur lui pour une fraction de seconde toute la lumière et la gloire, sur l'acier virginal... Seulement, vierge, il y avait belle lurette qu'elle ne l'était plus, [...]

Dans la suite du texte, les choses se compliquent cependant:

mais je suppose que ce n'était pas cela qu'il lui demandait espérait d'elle le jour où il avait décidé de l'épouser, sachant sans doute parfaitement dès ce moment ce qui l'attendait, ayant accepté par avance ayant assumé ayant par avance consommé si l'on peut dire cette Passion, avec cette différence que le lieu le centre l'autel n'en était pas une colline chauve, mais ce suave et tendre et vertigineux et broussailleux et secret repli de la chair... Ouais: crucifié, agonisant sur l'autel la bouche l'antre de..

Ici, il faudrait que, dans la traduction aussi, tous les mots se référant à la Passion se réfèrent à la fois au Christ (jamais nommé) et à de Reixach, au Golgotha (jamais nommé) et, par contraste, au sexe féminin: "une colline chauve " [...] «ce $[\ldots]$ broussailleux et secret repli de la chair».

Ici se pose aussi, dans toute son ampleur, la question des références culturelles et donc de la compétence en la matière des lecteurs et traducteurs. Pour Claude Simon, scolarisé dans un collège jésuite, tout cela allait de soi; je ne sais pas si pour de jeunes lecteurs français d'aujourd'hui ces références sont évidentes. Avec des étudiants norvégiens, j’ai vécu des moments ahurissants à propos de cette description. Et pour des lecteurs venus de cultures où l'histoire du Golgotha n'est pas fondatrice, je me demande ce que font les traducteurs... 


\section{LOST IN TRANSLATION}

À propos de la force génératrice des mots dans l'œuvre simonienne, je terminerai par quelques réflexions sur l'ouverture d'Histoire, la plus belle de toutes ses ouvertures, la plus belle et la plus intraduisible ${ }^{8}$. Il s'agit ici de l'incipit au sens strict du terme: l'une d'elles, et des conséquences de cette construction pronominale pour la construction même du roman:

l'une d'elles touchait presque la maison et l'été quand je travaillais tard dans la nuit assis devant la fenêtre ouverte je pouvais la voir ou du moins ses derniers rameaux éclairés par la lampe avec leurs feuilles semblables à des plumes palpitant faiblement sur le fond de ténèbres, les folioles ovales teintées d'un vert cru irréel par la lumière électrique remuant par moments comme des aigrettes comme animées soudain d'un mouvement propre (et derrière on pouvait percevoir se communiquant de proche en proche une mystérieuse et délicate rumeur invisible se propageant dans l'obscur fouillis des branches), comme si l'arbre tout entier se réveillait s'ébrouait se secouait, puis tout s'apaisait et elles reprenaient leur immobilité, les premières que frappaient directement les rayons de l'ampoule se détachant avec précision en avant des rameaux plus lointains de plus en plus faiblement éclairés de moins en moins distincts entrevus puis seulement devinés plus complètement invisibles quoiqu'on pût les sentir nombreux s'entrecroisant se succédant se superposant dans les épaisseurs d'obscurité d'où parvenaient de faibles froissements de faibles cris d'oiseaux endormis tressaillant s'agitant gémissant dans leur sommeil

Louis Hjelmslev, le linguiste danois, définit ainsi, 30 ans avant Benveniste, « la nature du pronom »:

Dans le pronom, il n'y a pas de représentation intuitive, il ne reste qu'une représentation in potentia, une unanschauliche Vorstellung /.../ Les particularités du pronom s'expliquent par ce fait évident que les mots appartenant à cette catégorie ne présentent aucun contenu significatif, aucun contenu "sémantique " dans le sens traditionnel du terme. Une simple observation des faits montre en effet que le seul contenu positif qu'on puisse trouver dans le pronom est celui que l'on retrouve dans les morphèmes?

C'est une définition qui décrit à merveille la pratique d'écriture de Simon dans Histoire. L'extraordinaire efficacité structurante de l'une d'elles est due à l'emploi optimal qu'il fait de cette forme pronominale vide pouvant représenter, sans se modifier, tous les objets au féminin, passant sans heurts du premier référent de elles, "branches ", au deuxième, au deuxième paragraphe, "les vieilles dames en visite/.../jacassant autour de grand-mère ». Et dans Histoire, l'une d'elles annonce en premier lieu la et les femmes qui ont peuplé la vie du narrateur.

8. Voir aussi Karin Holter, "L'UNE D'ELLES... OU L'HISTOIRE DES PREMIERS BATTEMENTS D'AILE D'HISTOIRE ", dans Isthmes francophones, du texte aux chants du monde. Mélanges offerts à Beïda Chikhi, Anne Douaire-Banny (dir.), "Lettres francophones », PUPS, 2012, p. 385-392.

9. Louis Hjelmslev, "La nature du pronom ", dans Mélanges offerts à P. van Ginneken (1937), republié dans Grand Larousse de la langue française, t..5, Paris, Larousse, 1976, p. 4676-4677. 
Mais il y a plus, et c'est ici que la traduction devient un art de l'impossible. Car, grâce à l'homophonie si caractéristique de la langue française, le morphème elle(s) a aussi un "contenu positif " - aile(s) - annonçant dès l'incipit la venue des oiseaux à la fin du premier paragraphe. Avec plumes et aigrettes qui annoncent aussi, déjà, les parures de la grand-mère et ses amies dans les paragraphes suivants. Et l'arbre lui-même, alias l'acacia, avec ses branches animées, devient très concrètement, " comme sur cette caricature orléaniste reproduite dans le manuel d'Histoire ", l'arbre généalogique de la famille. Voilà, comme le dit Beckett, pour le traducteur aussi " ce qu'il importe de ne pas perdre de vue, dans la chaleur de la rédaction $»$. 